\title{
HIGH PRECISION PHOTOMETRY OF 10,000 STARS IN M 3
}

R. Buonanno ${ }^{1}$, A. Buzzoni ${ }^{1}$, C. E. Corsi ${ }^{1}$, F. Fusi Pecci ${ }^{2}$ and A. R. Sandage ${ }^{3}$

Astronomical Observatory, Rome. 1 Department of Astronomy, Bologna. 2 Mt. Wilson and Las Campanas Obs. 3

ABSTRACT: A new color-magnitude diagram for $M 3$ is presented. 10,000 stars have been measured down to $\mathrm{V}=22$ with an internal accuracy better than $0.03 \mathrm{mag}$ to get complete and very accurate samples over well defined areas.

More than 10,000 stars have been measured down to $\mathrm{V}=22$ in two different areas. In the first, with $3.5<\mathrm{r}<6.0$ arcmin, photometric completeness has been achieved down to $\mathrm{V}=21.5$ and an algorithm to correct for losses due to unrecoverable crowding and blending has been experimentally computed. In the second, within a square field of $15 \mathrm{x}$ 15 arcmin, completeness has been extended only to $\mathrm{V}=18$, well below the horizontal branch.

Many tests made on the data guarantee an internal photometric accuracy better than $0.03 \mathrm{mag}$ at $\mathrm{V}=21$. Therefore, both the total population of each branch and the relative star-number ratios are "bona fide" representatives of the corresponding evolutionary time-scales. Here we simply present: 1) the color-magnitude diagram (see Fig. 1) obtained from the reduction of a wide collection of Palomar plates; 2) a table which presents the contribution of the various branches to the integrated cluster light; 3 ) the preliminary indication that, within the annulus we have considered, the blue stragglers seem to be slightly less centrally concentrated than the subgiants in the same magnitude interval. 


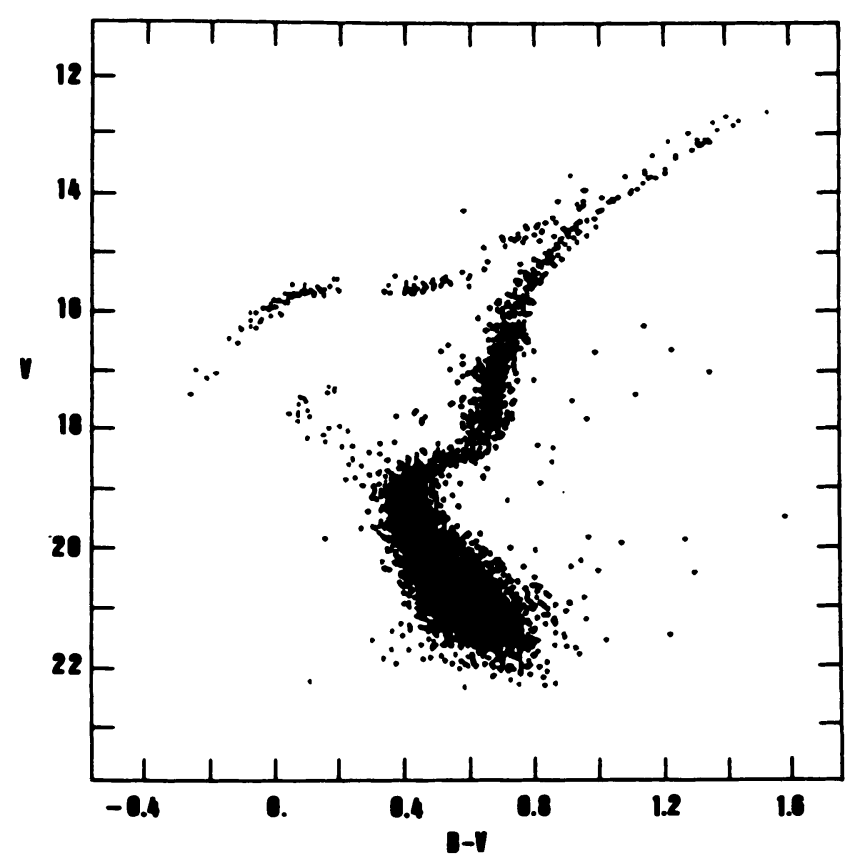

Fig. 1. CMD of M 3, composite sample.

\section{Table I:}

Contribution to the cluster integrated light of the various branches, computed over the complete samples

\begin{tabular}{|lrrrrr|}
\hline Phase & N(stars) & L/Lo & \%Bol & $\% \mathrm{~V}$ & $\% \mathrm{~B}$ \\
\hline AGB & 10 & 5524 & 19 & 11 & 7 \\
HB & 82 & 4178 & 14 & 17 & 25 \\
RGB & 342 & 10739 & 37 & 36 & 29 \\
SGB & 764 & 2228 & 8 & 10 & 11 \\
MS & 8628 & 5908 & 21 & 25 & 28 \\
BS & 53 & 193 & 0.7 & 1.0 & 1.3 \\
\hline
\end{tabular}

